May $20-24,1996$

16th International Cryogenic Engineering Conference / International Cryogenic Materials Conference and Industrial Exhibition

Kitakyushu, Japan

第16 回国際低温工学会議/国際低温材料会議

ICEC16 / ICMC

Abstract Booklet

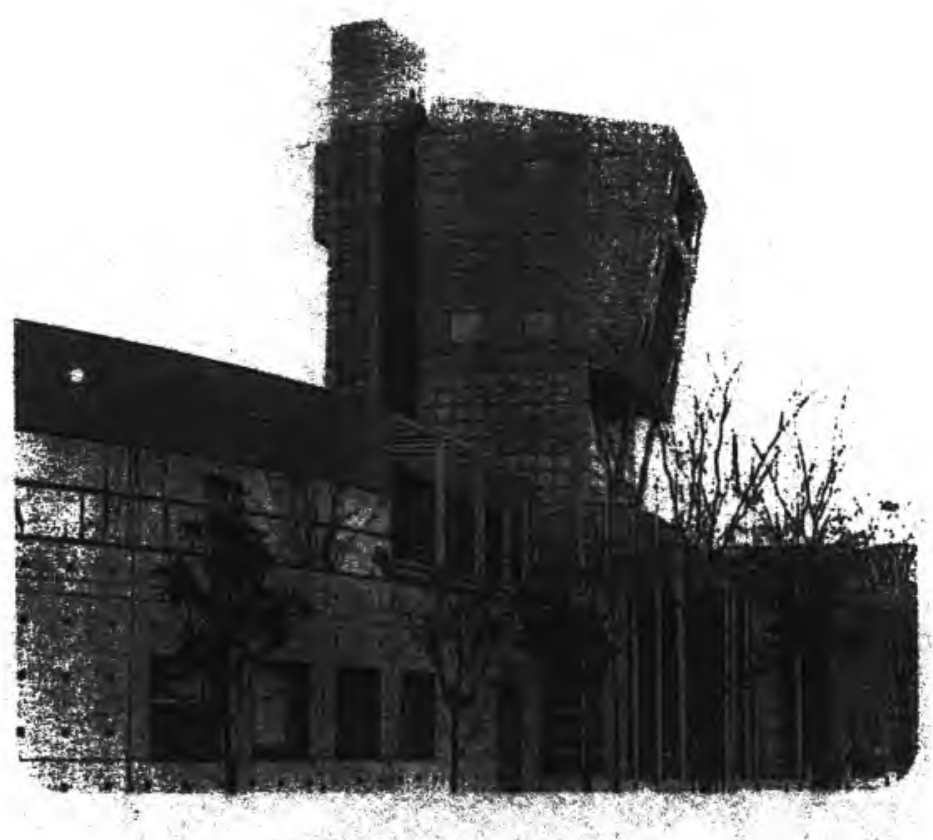




\title{
INHOMOGENEITY AND LOCAL NEGATIVE MAGNETORESISTANCE OF ALUMINUM
}

\author{
V:R.Sobol, O.N:Mazurenko, A.A.Drozd \\ (Institute of Physics of Solids and Semiconductors ASB, \\ P.Brovka Str. 17, Minsk 220072, Belarus)
}

The peculiarities of current distribution in aluminum plate samples have been investigated under the inhomogeneous action of transverse magnetic field at cryogenic temperatures. The mágnetic field inhomogeneity was arranged both along the transport direction and perpendicular to it with a method of bended sample being placed in external uniform field. The regime of current supply was used and the potential of steady electric field have been measured on the sample surface. The magnetic field gradient scales were up to $5 \mathrm{~T} / \mathrm{cm}$ and the spacial dependence of field on coordinates corresponded to the both linear and exponential law. For the case of longitudinal inhomogeneity there takes place a potential redistribution and at definite levels of magnetic field and its gradient a local negative magnetoresistance has been obseryed. For the transverse inhomogeneity the potential pattern is determined with local magnitude of magnetic field. The analysis of charge flow is being done with a help of continue equation and a rotate free type of electric field for the complex medium consisted of regions having monotonically changing hall coefficients. For the case when field has longitudinal gradient the current skinning and its allocation in hall direction occurs, transverse inhomogeneity being characterized with absence of hall charge flow. A definite analogy with composite conductors consisted of metals having different hall coefficients are being discussed. The noticed peculiarities in bended conductors should be taken into account in different superconductive cable composite stabilizers because current skinning and negativity of magnetoresistance lead to the appearance of self excessive magnetoresistance and heat generation in addition to that in interface region.

This research is supported by Fund of Fundamental Investigations of the Republic of Belarus (Grant No F40-249). 